TRANSACTIONS OF THE

AMERICAN MATHEMATICAL SOCIETY

Volume 348, Number 5, May 1996

\title{
ORIENTATION-REVERSING INVOLUTIONS ON HANDLEBODIES
}

\author{
JOHN KALLIONGIS AND DARRYL MCCULLOUGH
}

\begin{abstract}
The observation that the quotient orbifold of an orientationreversing involution on a 3-dimensional handlebody has the structure of a compression body leads to a strong classification theorem, and general structure theorems. The structure theorems decompose the action along invariant discs into actions on handlebodies which preserve the $I$-fibers of some $I$-bundle structure. As applications, various results of R. Nelson are proved without restrictive hypotheses.
\end{abstract}

\section{INTRODUCTION}

Throughout this paper $h$ will be an orientation-reversing involution on a 3dimensional orientable handlebody $H$. Moreover, $h$ is assumed to be tame, so the action of $h$ on an invariant ball about a fixed point must be equivalent either to the antipodal map on the 3-ball, or to a reflection across a 2-disc in the 3-ball, or (precisely when the fixed point lies in $\partial H$ ) to a reflection across a half 2-disc in a half 3-ball. The fixed point set fix $(h)$ is thus a union of finitely many isolated fixed points and finitely many 2 -manifolds properly imbedded in $H$. It is known (Theorem 6.1 of [2]) that the 2-dimensional components are incompressible, although this will be seen in passing when we examine the structure of $h$, and is immediate from the structure theory we will develop.

A simple type of involution occurs when $h$ is a product $h_{1} \times h_{2}$ on $A \times I$, where $A$ is an orientable 2-manifold with boundary, each $h_{i}$ is either the identity or an involution, and exactly one of the $h_{i}$ is orientation-reversing. Also, when $H$ is the orientable $I$-bundle over a nonorientable 2-manifold with boundary, one has similar examples. By gluing such actions together along invariant discs in the boundary, one constructs more complicated examples. Our main structure results, Theorems 5.1 and 5.6, show that all orientation-reversing involutions are built up in this way, and furnish descriptions of the component actions in terms of the fixed-point data of $h$ and the genus of $H$. The actions on the pieces in these decompositions are sometimes but not always unique up to equivalence; the conditions under which uniqueness holds and the extent to which it fails are fully analyzed in auxiliary theorems. As corollaries of the main structure results, one obtains the following simple characterizations of fiber-preserving involutions in terms of the fixed point data.

Received by the editors June 29, 1994 and, in revised form, May 4, 1995.

1991 Mathematics Subject Classification. Primary 57M60; Secondary 57S25.

Key words and phrases. 3-manifold, orbifold, handlebody, orientation-reversing, group action, involution, $I$-bundle, fiber-preserving, compression body, classification.

(C)1996 American Mathematical Society 
Corollary 5.8. Let $h$ be an orientation-reversing involution of a handlebody $H$. Then in some coordinates, $H$ is of the form $A \times I$ on which $h$ acts preserving $I$-fibers if and only if one of the following holds.

(i) $h$ is a vertical involution of a product I-bundle, or

(ii) every component of $\operatorname{fix}(h)$ is a disc or annulus, or

(iii) every component of $\operatorname{fix}(h)$ is a point.

Corollary 5.9. Let $h$ be an orientation-reversing involution of a handlebody $H$. Then in some coordinates, $H$ is an I-bundle on which $h$ acts preserving I-fibers if and only if one of the following holds.

(i) $h$ is a vertical involution of an I-bundle, or

(ii) every component of fix $(h)$ has nonnegative Euler characteristic.

The structure theory is actually rather transparent, given the description of the quotient orbifold $H / h$ that will be developed in $\S 2$ below. Using equivariant compressing discs, we will see that this quotient orbifold has a very restricted topological structure. We call it a silvered compression body. Such a structure is determined up to orbifold homeomorphism by the fixed point set and the genus of $H$, unless there are no isolated fixed points and every 2-dimensional component of the fixed point set is orientable, in which case the homeomorphism classes are determined by whether or not fix $(h)$ separates $H$. This translates into our Classification Theorem:

Theorem 3.6 (Classification Theorem). Let $h$ be an orientation-reversing involution on an orientable handlebody $H$. Then $h$ is determined up to equivalence by the homeomorphism type of $\operatorname{fix}(h)$, unless $\operatorname{fix}(h)$ is nonempty, each component of $\mathrm{fix}(h)$ is 2-dimensional and orientable, and the number of components of fix $(h)$ does not exceed $(\chi(\operatorname{fix}(h))-\chi(H)) / 2$. In this case, there are precisely two equivalence classes, determined by whether or not $\mathrm{fix}(h)$ separates $H$.

About a decade ago R. Nelson [10], [11] proved a number of results about such involutions, under the assumption that there are no isolated fixed points. These include special cases of several of our results, and we cite these at appropriate points in the paper. These motivated much of our work, although the methods are at least superficially different.

We use the language of orbifolds, as originated by Thurston [13] and expounded in [3]. Our arguments, however, rely very little on the theory of orbifolds; we are able to give all proofs using only elementary combinatorial topology.

Throughout the paper we will use $r$ to denote the reflection of an interval across its midpoint. Thus, if the interval is $I=[0,1], r(t)=1-t$, while if the interval is of the form $[-1,1], r(t)=-t$.

We thank St. Louis University for its support of the collaborative work of the authors.

\section{INVOLUTIONS OF $I$-BUNDLES}

Let $F$ be a compact connected 2-manifold. An involution of $F \times I$ is called a product action if it is of the form $h_{1} \times h_{2}$. Since every involution of $I$ is conjugate to $r$, any product involution is equivalent to one with $h_{2}$ equal to either $1_{I}$ or $r$.

Suppose that $H$ is an $I$-bundle over a compact 2-manifold $F$. If $F$ is nonorientable, let $\widetilde{F} \rightarrow F$ be the orientable double covering, with covering transformation $\tau$. We will regard $H$ as the quotient of $\widetilde{F} \times I$ or $\widetilde{F} \times[-1,1]$ by the free involution 
$\tau \times r$. Points of $H$ can then be denoted as $[x, t]$ where $x \in \widetilde{F}$. A product involution $h_{1} \times h_{2}$ of $\widetilde{F} \times I$ that commutes with $\tau \times r$ induces an involution on $H$, which will be denoted by $\left[h_{1} \times h_{2}\right]$. By the following result of Kim-Tollefson and Meeks-Scott, involutions that preserve the associated $\partial I$-bundle of $H$ are always equivalent to involutions of this form.

Proposition 1.1. Let $h$ be an orientation-reversing involution of a handlbody $H$, and suppose that $H$ has the structure of an I-bundle over a surface $F$, and $h$ preserves the associated $\partial I$-bundle. If $F$ is orientable, then $h$ is equivalent to an involution of the form $h_{1} \times h_{2}$, where $h_{2}$ is $1_{I}$ or $r$. If $F$ is nonorientable, then $h$ is conjugate to an involution of the form $\left[h_{1} \times 1_{I}\right]$.

Proof. If $F$ is orientable, this is immediate from Theorem A of [5] or Theorem 8.1 of [8]. Suppose $F$ is nonorientable. Let $\widetilde{F} \times I \rightarrow H$ be the two-fold covering by a product $I$-bundle. The lifts of $h$ to $\widetilde{F} \times I$ generate an action of $\mathbb{Z} / 2 \times \mathbb{Z} / 2$ on $\widetilde{F} \times I$ which preserves $\widetilde{F} \times \partial I$ (the lifts do not generate a cyclic group of order 4 , because $\tau \times r$ interchanges the components of $\partial \widetilde{F} \times I)$. By Theorem 8.1 of [8], the action of this group is conjugate to an action which preserves the product structure. This induces a conjugation of $h$ to an action of the form $\left[h_{1} \times 1_{I}\right]$.

In particular, Proposition 1.1 shows that every involution that is fiber-preserving with respect to some $I$-bundle structure is equivalent to one of the form $h_{1} \times h_{2}$ or $\left[h_{1} \times h_{2}\right]$.

An involution of $F \times I$ is said to be vertical if it is of the form $1_{F} \times r$, and horizontal if it is of the form $\sigma \times 1_{I}$, for some involution $\sigma$ of $F$. If $F$ is nonorientable, then an involution of the orientable $I$-bundle over $F$ is said to be vertical if it is of the form $\left[1_{\widetilde{F}} \times r\right]$. When $F$ is nonorientable, every fiber-preserving involution is considered to be horizontal, since by Proposition 1.1 it is conjugate to an involution of the form $\left[\sigma \times 1_{I}\right]$.

When $F$ is a disc, annulus, or Möbius band, a vertical action on $F \times I$ can also be regarded as a horizontal action for a different product structure. This observation is crucial to our decomposition theory, and is detailed in the proof of Theorem 5.1.

We close this section with some easy observations about fiber-preserving involutions, which will be used in our later arguments without further comment.

1 . Let $h$ be a vertical action on an $I$-bundle. Then the $I$-bundle is a product if and only if $\operatorname{fix}(h)$ is an orientable surface.

2. Let $h$ be a nonvertical orientation-reversing product action $h_{1} \times h_{2}$ on a product $I$-bundle $A \times I$, and suppose that fix $(h)$ is nonempty. Then fix $(h)$ consists of isolated points if and only if $h_{1}$ is orientation-preserving, and consists of a collection of discs and annuli if and only if $h_{1}$ is orientation-reversing.

3. Let $h$ be a nonvertical orientation-reversing fiber-preserving action on an orientable $I$-bundle over a nonorientable surface. Then every component of fix $(h)$ is a point, a disc, an annulus, or a Möbius band.

\section{Silvered COMPRESSION BODIES AND THE STANDARD CONSTRUCTION}

Adapting a definition due to Bonahon [1] and McCullough and Miller [6], we call a 3-orbifold $V$ a silvered compression body when it can be constructed as follows. Start with a collection $\left\{F_{i} \mid 1 \leq i \leq m\right\}$ of connected 2-manifolds, each having nonempty boundary, and a collection $\left\{W_{j} \mid 1 \leq j \leq n\right\}$ of 3-orbifolds each of which 
is the quotient of the 3 -ball $D^{3}$ by the antipodal involution. Each $W_{j}$ is topologically the cone on a projective plane which we will denote by $P_{j}$, and the cone point $p_{j}$ is singular with local group of order 2 . Form a connected irreducible 3-orbifold $V$ from $\left(\bigcup_{i=1}^{m} F_{i} \times I\right) \cup\left(\bigcup_{j=1}^{n} W_{j}\right)$ by attaching $k$ 1-handles to $\left(\bigcup_{i=1}^{m} F_{i} \times\{1\}\right) \cup\left(\bigcup_{j=1}^{n} P_{j}\right)$. Here, $m+n \geq 1$ and $k \geq m+n-1$. Regard each point of $\bigcup_{i=1}^{m} F_{i} \times\{0\}$ as a mirror point. More precisely, let $D^{3}$ be the standard 3-ball in $\mathbb{R}^{3}$, and let $D_{+}^{3}$ denote the points in $D^{3}$ having nonnegative $z$-coordinate. Let $\rho: D^{3} \rightarrow D^{3}$ be the involution defined by $\rho(x, y, z)=(-x, y, z)$. Then each point $w$ of $F_{i} \times\{0\}$ has an orbifold chart $(U, w)=\left(D^{3} / \rho,(0,0,0)\right)$ or $(U, w)=\left(D_{+}^{3} / \rho,(0,0,0)\right)$ according as $w$ does or does not lie in $\partial F_{i} \times\{0\}$. We denote $F_{i} \times\{0\}$ by $F_{i}$. Note that the (orbifold) boundary of $V$ is connected.

Lemma 2.1. Let $h$ be an orientation-reversing involution on a handlebody $H$. Then the quotient orbifold $H / h$ is either a silvered compression body or a nonorientable handlebody.

Proof. We induct on the genus of $H$. If the genus is 0 then by conjugation $H$ may be regarded as $D^{3}$, on which either $h(x, y, z)=(-x, y, z)$ or $h(x, y, z)=(-x,-y,-z)$. In the first case, the quotient is a silvered compression body with $m=1, F_{1}=D^{2}$, and $n=k=0$. In the second case, it is a silvered compression body with $m=k=0$ and $n=1$.

Assume that the genus of $H$ is positive. By the Equivariant Loop Theorem [9], or by [4], there exists an essential compressing disc $D$ such that either $h(D)=D$ or $h(D)$ is disjoint from $D$. If $D$ lies in fix $(h)$, or if $D \cap$ fix $(h)$ consists of a single point, we can replace it with the boundary of an equivariant product neighborhood of $D$. Therefore we may assume that either $D \cap \operatorname{fix}(h)$ is empty or $D \cap \operatorname{fix}(h)$ is an arc. Then, either (i) $D$ is disjoint from $h(D)$ and $D \cup h(D)) / h$ is a disc disjoint from the singular set of $H / h$, or (ii) $h(D)=D$ and $D / h$ is a square with one side silvered. In case (i), if $D \cup h(D)$ separates $H$ into two components and $h$ interchanges the complementary components, then $h$ acts freely, so $H / h$ is a nonorientable handlebody. Suppose that $D \cup h(D)$ separates $H$ into three components with $h$ interchanging two of the components and leaving the third, $H_{1}$, invariant. Since $H_{1}$ is a handlebody of lower genus than $H, H_{1} / h$ is a silvered compression body. Since $H / h$ is obtained from $H_{1} / h$ by attaching 1-handles, it is a silvered compression body. Suppose that either $D \cup h(D)$ separates $H$ and none of the complementary components are exchanged, or $D \cup h(D)$ does not separate $H$. Each component of the orbifold that results from cutting $H / h$ along $(D \cup h(D)) / h$ is the quotient of an orientation-reversing involution on a component of the handlebody that results from cutting $H$ along $D \cup h(D)$. Such a component must be a handlebody of lower genus than $H$, so each component of $H / h$ cut along $(D \cup h(D)) / h$ is a silvered compression body. Therefore $H / h$ is a silvered compession body as well. In case (ii), each component of $H$ cut along $D$ is invariant under $h$, so by induction has quotient a silvered compression body. The image of $D / h$ in each of these lies in the boundary and meets the silvered part in its silvered edge, so by isotopy we may asssume that the copies of $D / h$ are of the form $\gamma \times I \subset F_{i} \times I$ for some arc $\gamma$ in $\partial F_{i}$. This makes the silvered compression body structure of $H / h$ clear, completing the induction.

Remark 2.2. Orientation-preserving group actions on handlebodies were extensively studied in [7] using orbifold techniques. To extend some of that approach to actions 
with orientation-reversing elements, one could define silvered orbifold compression bodies using arbitrary 2-orbifolds with boundary in place of the $F_{i}$ and arbitrary discal 3-orbifolds in place of the $W_{i}$, and attaching them together using orbifold 1-handles of the form $D \times I$ where $D$ is a discal 2-orbifold. Then, the Equivariant Loop Theorem shows that the quotient of an arbitrary tame action of a finite group on a handlebody is a handlebody or a silvered orbifold compression body. For orientation-reversing involutions we will have no need of this level of generality, so we have used the simpler specialized definition given above.

There is a concept of orbifold fundamental group, and an orbifold covering space theory analogous to the usual theory. In [13] there is a construction of the universal covering orbifold, and the orbifold fundamental group is by definition the covering transformations for this covering. Each connected nonorientable 3 -orbifold has a unique double covering by an orientable orbifold. A quick construction of the orientable double covering of a silvered compression body can be given as follows: (1) take the orientable double covering of the underlying manifold of $V-\left\{p_{1}, \ldots, p_{n}\right\}$ (or take two homeomorphic copies of it interchanged by a covering involution, if the underlying manifold is orientable), (2) form its quotient space by identifying points in the preimage of $\bigcup_{i=1}^{m} F_{i}$ which are translates under the covering involution, and (3) add in single points which compactify each of the $S^{2} \times[0,1)$ ends; these will map to the $p_{j}$ under the covering. The manifold covering involution induces the orbifold covering involution.

For our later arguments, we will need a very explicit description of the structure of the silvered compression body and its double covering (from which the critically important uniqueness of this covering is seen without any appeal to orbifold theory). We will now describe a standard construction for a silvered compression body $V$ having $k$ 1-handles. The construction will produce a silvered compression body in each orbifold homeomorphism class. Its structure is uniquely defined within this class, except for the choice of which 2-dimensional component of fix $(h)$ is denoted by $F_{1}$. The notation that describes the various pieces in the construction will be used throughout the rest of the paper.

Definition 2.3. A silvered compression body is called mirrorless if $m=0$, coneless if $n=0$, and exceptional if it is coneless, all $F_{i}$ are orientable, and its underlying topological space is nonorientable.

Suppose first that $V$ is mirrorless. In $P_{1}$, fix disjoint imbedded discs $E$ and $E_{1}$, in $P_{j}$ for $2 \leq j \leq n-1$ fix disjoint imbedded discs $E_{j}^{\prime}$ and $E_{j}$, and fix an imbedded $\operatorname{disc} E_{n}^{\prime}$ in $P_{n}$. Fix $2(k+1-n)$ discs $E_{j, 1}, E_{j, 2}, n \leq j \leq k$, disjointly imbedded in $E$. For $1 \leq j \leq n-1$, let $L_{j}=D^{1} \times D^{2}$ be a 1-handle with $\{0\} \times D^{2}$ identified with $E_{j}$ and $\{1\} \times D^{2}$ identified with $E_{j+1}^{\prime}$. For $n \leq j \leq k$, let $L_{j}=D^{1} \times D^{2}$ be a 1-handle with $\{0\} \times D^{2}$ identified with $E_{j, 1}$ and $\{1\} \times D^{2}$ identified with $E_{j, 2}$. The latter identifications are to be performed so that if $N$ is a regular neighborhood of $E$ in $W_{1}$, then $N \cup\left(\bigcup_{j=n}^{k} L_{j}\right)$ is an orientable handlebody (of genus $k+1-n$ ). Note that we may always choose the compression body structure in this way; if $N \cup\left(\bigcup_{j=n}^{k} L_{j}\right)$ is nonorientable, then since $P_{1}$ is nonorientable, $E$ may be reselected to make $N \cup\left(\bigcup_{j=n}^{k} L_{j}\right)$ orientable.

Suppose now that $V$ is coneless. If any $F_{i}$ is nonorientable, renumber the $F_{i}$ if necessary to ensure that $F_{1}$ is nonorientable. In $F_{1} \times\{1\}$, fix disjoint imbedded 
discs $D$ and $D_{1}$, in $F_{i} \times\{1\}$ for $2 \leq i \leq m-1$ fix disjoint imbedded discs $D_{i}^{\prime}$ and $D_{i}$, and fix an imbedded disc $D_{m}^{\prime}$ in $F_{m} \times\{1\}$. Fix $2(k+1-m) \operatorname{discs} D_{i, 1}, D_{i, 2}$, $m \leq i \leq k$, disjointly imbedded in $D$. For $1 \leq i \leq m-1$, let $K_{i}=D^{1} \times D^{2}$ be a 1-handle with $\{0\} \times D^{2}$ identified with $D_{i}$ and $\{1\} \times D^{2}$ identified with $D_{i+1}^{\prime}$. For $m \leq i \leq k$, let $K_{i}=D^{1} \times D^{2}$ be a 1-handle with $\{0\} \times D^{2}$ identified with $D_{i, 1}$ and $\{1\} \times \bar{D}^{2}$ identified with $D_{i, 2}$.

If $V$ is coneless but nonexceptional, then as for the mirrorless case the latter identifications are to be performed so that if $N$ is a regular neighborhood of $D$ in $F_{1} \times I$, then $N \cup\left(\bigcup_{i=m}^{k} K_{i}\right)$ is an orientable handlebody (of genus $k+1-m$ ). If $V$ is exceptional, then this will not be possible. In this case carry out the attachings of the $K_{i}$ so that $K_{m}$ reverses orientation in $N \cup\left(\bigcup_{i=m}^{k} K_{i}\right)$ but $K_{m+1}, \ldots, K_{k}$ preserve orientation. Since any nonorientable handlebody is homeomorphic to a ball with 1-handles attached, all but one of which are orientation-preserving, the standard structure can always be selected as we have described.

If $V$ is neither coneless nor mirrorless, we omit $E$, and the $E_{j, 1}$ and $E_{j, 2}$ for $j \geq n$, but instead select $D_{m}$ and $E_{1}^{\prime}$ in $F_{m} \times\{1\}$ and $P_{1}$ respectively, and add an additional 1-handle $L_{n}$ that connects $D_{m}$ to $E_{1}^{\prime}$. The 1-handles of $V$ are $K_{1}, \ldots$, $K_{k-n}, L_{1}, \ldots, L_{n}$.

We can now prove

Lemma 2.4. Let $V$ be a nonorientable handlebody or a silvered compression body. Then $V$ has an orbifold double covering $H \rightarrow V$ where $H$ is an orientable handlebody. Any other orbifold double covering by an orientable manifold is equivalent to this one.

Proof. For a nonorientable handlebody, $H$ is just the manifold double covering. Suppose $V$ is a silvered compression body. First we examine the double coverings of the pieces of $V$. Consider an orbifold $F_{i} \times I$ with $F_{i} \times\{0\}$ silvered. If $F_{i}$ is orientable, this orbifold has a unique 2 -fold covering by a manifold, namely $F_{i} \times[-1,1]$, with covering transformation sending $(x, t)$ to $(x,-t)$. If $F_{i}$ is nonorientable, then there will be more than one covering by a manifold. Each covering is an $I$-bundle over $F_{i}$, and among them is the unique $I$-bundle over $F_{i}$ with orientable total space. Finally, each $W_{i}$ has a unique double covering by a manifold, which is $D^{3}$ with covering transformation the antipodal map. Denote the covering of $F_{i} \times I$ by an orientable $I$-bundle by $p_{i}: X_{i} \rightarrow F_{i} \times I$, and the covering of $W_{j}$ by $D^{3}$ by $q_{j}: Y_{j} \rightarrow W_{j}$. Denote the orbifold covering transformations by $\sigma_{i}$ for the $X_{i}$ and $\tau_{j}$ for the $Y_{j}$. These are orientation-reversing.

Suppose first that $V$ is coneless. Let $H_{0}=\bigcup_{i=1}^{m} X_{i}$, and let $h_{0}$ be the involution that is $\sigma_{i}$ on each $X_{i}$. Fix orientations for the $X_{i}$, and for the discs $D$ and $D_{i}$. Orient the 1-handles $K_{i}=D^{1} \times D^{2}$ for $1 \leq i \leq m-1$ (thus determining orientations for $\{0\} \times D^{2}$ and $\{1\} \times D^{2}$ as submanifolds of $\partial K_{i}$ ) so that the identification of $\{0\} \times D^{2}$ with $D_{i}$ preserves orientation. Then, orient the $D_{i}^{\prime}$ so that the identification of $\{1\} \times D^{2}$ with $D_{i+1}^{\prime}$ preserves orientation. Each of the discs $D, D_{i}$, and $D_{i}^{\prime}$ has a pair of lifts to $H_{0}$; let $\widetilde{D}$ be the one whose lifted orientation agrees with that of $\partial X_{1}$, and similarly determine $\widetilde{D_{i}}$ and $\widetilde{D_{i}^{\prime}}$. The other lifts are $h_{0}(\widetilde{D})$, and so on. Now connect each $\widetilde{D_{i}}$ to $\widetilde{D_{i+1}^{\prime}}$ by a 1 -handle, which we denote by $\widetilde{K_{i}}$, so that the identifications of $\widetilde{D_{i}}$ and $\widetilde{D_{i+1}^{\prime}}$ with the discs in $\partial \widetilde{K_{i}}$ are orientation-preserving. Attach additional 1-handles connecting $h_{0}\left(\widetilde{D_{i}}\right)$ and $h_{0}\left(\widetilde{D_{i+1}^{\prime}}\right)$ so that the resulting handlebody $H_{1}$ is 
orientable. Our orientation choices have ensured that the orbifold covering $H_{0} \rightarrow$ $\bigcup_{i=1}^{m} F_{i} \times I$ extends to a covering $H_{1} \rightarrow\left(\bigcup_{i=1}^{m} F_{i} \times I\right) \cup\left(\bigcup_{i=1}^{m-1} K_{i}\right)$, whose covering involution $h_{1}$ restricts to $h_{0}$ on $H_{0}$. Now suppose that $V$ is nonexceptional, so that a regular neighborhood of $D \cup\left(\bigcup_{i=m}^{k} K_{i}\right)$ is orientable. For $m \leq i \leq k$, let $\widetilde{D_{i, 1}}$ and $\widetilde{D_{i, 2}}$ be the lifts of $D_{i, 1}$ and $D_{i, 2}$ that lie in $\widetilde{D}$. Attach 1-handles $\widetilde{K}_{i}$ connecting $\widetilde{D_{i, 1}}$ and $\widetilde{D_{i, 2}}$ so that the union of these 1-handles with a regular neighborhood of $\widetilde{D}$ is an orientable handlebody. Attach corresponding 1-handles which connect $h_{1}\left(\widetilde{D_{i, 1}}\right)$ and $h_{1}\left(\widetilde{D_{i, 2}}\right)$. The result is an orientable handlebody $H$, so that $h_{1}$ extends to an involution $h$ whose quotient orbifold is $V$. If $V$ is exceptional, however, then $\widetilde{K_{m}}$ must be attached to $\widetilde{D_{m, 1}}$ and $h_{1}\left(\widetilde{D_{m, 2}}\right)$, in order that the covering map extend to $\widetilde{K_{m}}$.

For the uniqueness, note first that any orientable orbifold double covering by a manifold must contain preimages of the $F_{i} \times I$ which are $X_{i}$. Given a double covering, define $h_{0}$ and the lifts $\widetilde{D}, \widetilde{D_{i}}$, and $\widetilde{D}_{i}^{\prime}$ as in the previous paragraph. If a lift of $K_{i}$ connects $\widetilde{D_{i}}$ to $h_{0}\left(\widetilde{D_{i+1}^{\prime}}\right)$, or $\widetilde{D_{i, 1}}$ to $h_{0}\left(\widetilde{D_{i, 2}}\right)$, for $i \neq m$, then the covering manifold is nonorientable. To make the covering manifold orientable, the lift of $K_{m}$ must connect $\widetilde{D_{m, 1}}$ to $\widetilde{D_{m, 2}}$, when $V$ is nonexceptional, and $\widetilde{D_{m, 1}}$ to $h\left(\widetilde{D_{m, 2}}\right)$, when $V$ is exceptional. Therefore the handlebody $H$ previously constructed is the only covering by an orientable manifold.

The cases when $V$ is mirrorless, or neither coneless nor mirrorless, are similar but easier, since in all cases a lift of $K_{m}$ connects $\widetilde{D_{m, 1}}$ to $\widetilde{D_{m, 2}}$.

Definition 2.5. The covering $H \rightarrow V$ constructed in Lemma 2.4 is called the orientable double covering of $V$.

Remark 2.6. Note that in the exceptional case, the preimage of $\bigcup_{i=1}^{m} F_{i}$ separates the preimage of $V-K_{m}$ into two components, but the lifts of $K_{m}$ connect the complementary components so that the preimage of $\bigcup_{i=1}^{m} F_{i}$ does not separate $H$. If $V$ is coneless and all $F_{i}$ are orientable, but $V$ is nonexceptional (i. e. the underlying manifold of $V$ is orientable), then the preimage of $\bigcup_{i=1}^{m} F_{i}$ separates $H$ and the complement has exactly two components. When $V$ is not coneless, or some $F_{i}$ is nonorientable, or $V$ is exceptional, the preimage does not separate.

\section{The Riemann-Hurwitz Formula and the Classification Theorem}

Let $g$ be the genus of $H$. The Euler characteristic of $H$ is $1-g$, while from the description of the orientable double cover of $H / h$ in $\S 2$, we have

$$
\chi(H)=\sum_{i=1}^{m} \chi\left(X_{i}\right)+n-2 k .
$$

Since $\chi\left(X_{i}\right)=\chi\left(F_{i}\right)$, we deduce

Theorem 3.1 (Riemann-Hurwitz Formula). Let $h$ be an orientation-reversing involution on an orientable handlebody $H$ of genus $g$, and suppose the fixed-point set of $h$ has $n$ isolated fixed points and has 2-dimensional components $F_{1}, \ldots, F_{m}$, and let $k$ be the number of 1 -handles in the silvered compression body $H / h$. Then

$$
g=2 k+1-n-\sum_{i=1}^{m} \chi\left(F_{i}\right) .
$$


This formula can also be stated as $2 k=\chi(\operatorname{fix}(h))-\chi(H)$. Since the number of components of $\operatorname{fix}(h)$ is $m+n$, and $k \geq m+n-1$, this leads to an easy corollary.

Corollary 3.2. Let $h$ be an orientation-reversing involution on an orientable handlebody $H$. Then $\operatorname{fix}(h)$ has at most $(\chi(\operatorname{fix}(h))-\chi(H)) / 2+1$ components.

Since $\chi(\operatorname{fix}(h))$ cannot exceed the number of components of fix $(h)$, the formula in Corollary 3.2 yields

Corollary 3.3. Let $h$ be an orientation-reversing involution on an orientable handlebody $H$ of genus $g$. Then the number of components of $\operatorname{fix}(h)$ is at most $g+1$.

Notation 3.4. If $V$ is a silvered compression body, let $|V|$ denote the manifold obtained by removing the cone points $\left\{p_{1}, \ldots, p_{n}\right\}$ from the underlying topological space of $V$.

We first deduce a preliminary version of the Classification Theorem.

Proposition 3.5. Let $h_{1}$ and $h_{2}$ be orientation-reversing involutions on an orientable handlebody $H$. Then $h_{1}$ and $h_{2}$ are equivalent if and only if $\operatorname{fix}\left(h_{1}\right)$ is homeomorphic to $\operatorname{fix}\left(h_{2}\right)$ and $\left|H / h_{1}\right|$ and $\left|H / h_{2}\right|$ are both orientable or both nonorientable.

Proof. One direction is trivial. For the other, assume that fix $\left(h_{1}\right)$ is homeomorphic to fix $\left(h_{2}\right)$. If these fixed-point sets are empty, then $H / h_{1}$ and $H / h_{2}$ are nonorientable handlebodies with the same Euler characteristic, so are homeomorphic. A homeomorphism between them lifts to an equivariant homeomorphism of the orientable double covers, which are $H$, and hence determines an equivalence of $h_{1}$ and $h_{2}$. Suppose the fixed-point sets are nonempty. By Lemma $2.1, H / h_{1}$ and $H / h_{2}$ are silvered compression bodies, with homeomorphic silvered set and the same number of cone points. From the Riemann-Hurwitz Formula, they have the same number of 1-handles. Considering the standard construction in $\S 2$, it is clear that they are homeomorphic unless they are coneless and one is exceptional and the other is not, in which case there are two homeomorphism classes determined by the orientability of the underlying manifold. An orbifold homeomorphism from $H / h_{1}$ to $H / h_{2}$ lifts to homeomorphisms from the orbifold coverings of $H / h_{1}$ to those of $H / h_{2}$. In particular, it must lift to an equivariant homeomorphism between the unique covers that are orientable manifolds, and hence it determines an equivalence between $h_{1}$ and $h_{2}$.

We can now prove one of our main results.

Theorem 3.6 (Classification Theorem). Let $h$ be an orientation-reversing involution on an orientable handlebody $H$. Then $h$ is determined up to equivalence by the homeomorphism type of fix $(h)$, unless fix $(h)$ is nonempty, each component of $\operatorname{fix}(h)$ is 2-dimensional and orientable, and the number of components of fix $(h)$ does not exceed $(\chi(\operatorname{fix}(h))-\chi(H)) / 2$. In this case, there are precisely two equivalence classes, determined by whether or not fix $(h)$ separates $H$.

Proof. If fix $(h)$ is empty, or contains an isolated point, or has a nonorientable 2dimensional component, then $|H / h|$ is nonorientable and Proposition 3.5 applies. If $\operatorname{fix}(h)$ is nonempty, every component of $\operatorname{fix}(h)$ is 2-dimensional and orientable, and the number of component of fix $(h)$ exceeds $(\chi(\operatorname{fix}(h))-\chi(H)) / 2$, then we have 
$k+1 \geq m>(\chi(\operatorname{fix}(h))-\chi(H)) / 2=k$, so $k=m-1$ and $|H / h|$ is constructed by connecting $m$ orientable manifolds $F_{i} \times I$ using $m-1$ handles, and hence it must be orientable. Again, by Proposition 3.5, $h$ is determined up to equivalence. In the remaining cases, we have $n=0$ and $m \leq k$, so $|H / h|$ can be orientable or not according to how the 1-handles $K_{m}, \ldots, K_{k}$ are attached. By Remark 2.6, these two possibilities correspond to whether or not fix $(h)$ separates $H$.

Remark 3.7. The Riemann-Hurwitz Formula and the Classification Theorem could be used to develop an enumeration of the orientation-reversing involutions on a handlebody of fixed genus $g$. Such an enumeration has been obtained using more group-theoretic methods by M. Stephanus [12].

\section{Actions With Empty or 0-Dimensional Fixed POINT SET}

In preparation for the structure theorems of the next section, we will analyze the special cases when the fixed-point set of $h$ is empty or 0-dimensional. By the Classification Theorem, such actions are detemined up to equivalence by the number of fixed points.

Proposition 4.1. Let $h$ be a free orientation-reversing involution on a handlebody $H$. Then $H$ is homeomorphic to a product $A \times I$ on which the action of $h$ is horizontal.

Proof. From the Lefschetz Fixed Point Formula, the genus of $H$ must be odd. Since $h$ is determined up to equivalence, we may choose any explicit realization of it. Let $h_{1}$ be the free involution on the solid torus $S^{1} \times I \times I$ given by $h_{1}(\theta, s, t)=$ $(\theta+\pi, s, 1-t)$, where we regard $S^{1}$ as $\mathbb{R} /\langle 2 \pi\rangle$. Letting $A=S^{1} \times\{1 / 2\} \times I$, we can regard the solid torus as $A \times I$, so that $h_{1}$ is horizontal. This completes the case when the genus of $H$ is 1 . If the genus is $1+2 k$, choose discs $D_{1,1}, D_{1,2}$, $D_{2,1}, \ldots, D_{k, 2}$ in $S^{1} \times I \times\{1\}$, which meet $A$ in proper arcs, and attach 1-handles $H_{i}, 1 \leq i \leq k$, which connect $D_{i, 1}$ to $D_{i, 2}$. For $1 \leq i \leq k$, let $a_{i}$ be a band in $H_{i}$ which connects $A \cap D_{i, 1}$ to $A \cap D_{i, 2}$, so that $A \cup a_{i}$ is orientable. Attach corresponding handles $H_{i}^{\prime}$ connecting $h_{1}\left(D_{i, 1}\right)$ to $h_{1}\left(D_{i, 2}\right)$, and extend $h_{1}$ to an involution $h$ on the union $(A \times I) \cup\left(\bigcup_{i=1}^{k} H_{i}\right) \cup\left(\bigcup_{i=1}^{k} H_{i}^{\prime}\right)$ which is horizontal with respect to a product structure $\left(\left(A \cup\left(\bigcup_{i=1}^{k} a_{i}\right) \cup\left(\bigcup_{i=1}^{k} h\left(a_{i}\right)\right)\right) \times I\right.$. This completes the proof.

Suppose now that $H$ has genus $g$ and fix $(h)$ contains $n$ isolated fixed points, where $n \geq g+1$. By Corollary 3.3, fix $(h)$ must consists of exactly $g+1$ fixed points. Thus $H / h$ is mirrorless and consists of $\bigcup_{j=1}^{g+1} W_{j}$ connected by 1-handles $L_{j}, 1 \leq j \leq g$. We will refine the standard construction of $H / h$ from $\S 2$. In each 3-ball $Y_{j}$ in $H$, fix a properly imbedded 2-disc $A_{j}$ invariant under $h_{0}$. Its image in $W_{j}$ is an orbifold $a_{j}$ whose underlying manifold is a 2-disc, and has one order 2 cone point. We require that each disc $E_{j}$ or $E_{j}^{\prime}$ meet $\partial a_{j}$ in an arc properly imbedded in $E_{j}$ or $E_{j}^{\prime}$. In $L_{j}$ fix a band $b_{j}=D^{1} \times D^{1} \subseteq D^{1} \times D^{2}$ whose ends $\partial D^{1} \times D^{1}$ connect the arcs $E_{j} \cap \partial a_{j}$ and $E_{j+1}^{\prime} \cap \partial a_{j+1}$. Let $B_{j}$ be a component of the preimage of $b_{j}$. Now $\left(\bigcup_{j=1}^{g+1} a_{j}\right) \cup\left(\bigcup_{j=1}^{g} b_{j}\right)$ is a 2-orbifold $B$ whose preimage in $H$ is a surface $S_{g+1}=\left(\bigcup_{j=1}^{g+1} A_{j}\right) \cup\left(\bigcup_{j=1}^{g} B_{j}\right) \cup\left(\bigcup_{j=1}^{g} h\left(B_{j}\right)\right)$. Let $\sigma$ be the restriction of $h$ to $S_{g+1}$; it rotates each disc $A_{j}$ about its center and interchanges each pair of bands $B_{j}$ and $h\left(B_{j}\right)$. The restriction of $p$ to $S_{g+1}$ is the unique 2 -fold branched covering 
of the 2-disc branched over the $g+1$ points $\left\{p_{1}, \ldots, p_{g+1}\right\}$. Therefore the Euler characteristic $\chi\left(S_{g+1}\right)$ is $1-g$, and $S_{g+1}$ has one boundary circle if $g$ is even and two boundary circles if $g$ is odd. Observe that $H$ can be regarded as a product $S_{g+1} \times[-1,1]$ such that $h$ is of the form $\sigma \times r$.

Next suppose that fix $(h)$ is 0 -dimensional and consists of $n$ isolated points, where $1 \leq n \leq g+1$. From the Riemann-Hurwitz Formula we now have $2 k=g+n-1$, so $g+1-n$ must be even, say $g+1-n=2 \ell$. As in the previous paragraph, there is an orientation-reversing involution $\sigma \times r$ with $n$ isolated fixed points in $S_{n} \times[-1,1]$, where $\chi\left(S_{n}\right)=2-n$ and $S_{n}$ has one boundary circle if $n$ is odd and two boundary circles if $n$ is even. Let $T$ be a disc-with- $\ell$-holes, and $t$ an arc in its boundary, and form $S_{n, \ell}$ by identifying the copies of $t$ in two copies of $T$ to two disjoint arcs in $\partial S_{n}$ which are interchanged by $\sigma$. Extend $\sigma$ to an involution $\sigma$ on $S_{n, \ell}$ so that $h$ is of the form $\sigma \times r$ on $S_{n, \ell} \times[-1,1]$. In summary, we have the following structure theorem for actions with 0 -dimensional fixed point set.

Theorem 4.2. Let $h$ be an orientation-reversing involution of an orientable handlebody $H$ of genus $g$, and suppose that $\operatorname{fix}(h)$ consists of $n$ isolated fixed points, where $n \geq 1$. Then $g+1-n$ is even, and $H$ has coordinates $S_{n,(g+1-n) / 2} \times[-1,1]$ on which $h$ acts as $\sigma \times r$.

\section{Structure theory}

There are several reasonable ways to split an orientation-reversing involution on a handlebody along invariant discs into simpler actions, specifically, into actions which are fiber-preserving with respect to some $I$-bundle structure. The ones that appear to be most useful are called the vertical-horizontal decomposition and the product decomposition. In each of them, the actions on the pieces that result from the splitting are often but not always uniquely determined up to equivalence; we will give precise uniqueness statements as auxiliary theorems. We will discuss other types of decompositions at the end of this section.

We first treat the vertical-horizontal decomposition.

Theorem 5.1 (Vertical-horizontal decomposition). Let $h$ be an orientationreversing involution of a handlebody $H$, and suppose that some component of fix $(h)$ is 2-dimensional. Then $H$ has a decomposition $H=H_{0} \cup\left(\bigcup_{j=1}^{r} H_{j}\right)$, where each piece is h-invariant, such that

(i) $H_{0}$ is an I-bundle over a connected 2-manifold, and the restriction of $h$ to $H_{0}$ is horizontal. This action may be chosen to be a product action if and only if no component of fix $(h)$ is a point or a Möbius band.

(ii) Each $H_{j}$ is an I-bundle over a surface of negative Euler characteristic, which is a deformation retract of a component of $\operatorname{fix}(h)$, and the restriction of $h$ to $H_{j}$ is vertical.

(iii) $\left\{H_{1}, \ldots, H_{r}\right\}$ are pairwise disjoint, and for $1 \leq i \leq r$ each $H_{0} \cap H_{i}$ is a single 2-disc.

Theorem 5.2. Let $h$ be an orientation-reversing involution of a handlebody $H$, and suppose that some component of $\operatorname{fix}(h)$ is 2 -dimensional. In any vertical-horizontal decomposition

$$
H=H_{0} \cup\left(\bigcup_{j=1}^{r} H_{j}\right)
$$


satisfying conditions (i), (ii), and (iii) in Theorem 5.1, the actions on the $H_{j}$ for $j \geq 1$ are determined up to equivalence (and ordering of the indices). The action on $H_{0}$ is determined up to equivalence if any of the following holds:

1. Every component of fix $(h)$ has nonnegative Euler characteristic.

2. Every component of $\mathrm{fix}(h)$ is 2-dimensional and orientable.

3. Some component of fix $(h)$ is a point or a Möbius band.

4. The number of components of fix $(h)$ exceeds $(\chi(\mathrm{fix}(h))-\chi(H)) / 2$.

In the remaining cases, either of two possible actions on $H_{0}$ can appear in verticalhorizontal decompositions of $h$.

Remark 5.3. We recall from Corollary 3.2 that the number of components of the fixed point set is at most $(\chi(\operatorname{fix}(h))-\chi(H)) / 2+1$. Thus condition 4 holds exactly when the number of components of fix $(h)$ equals $(\chi(\operatorname{fix}(h))-\chi(H)) / 2+1$.

Remark 5.4. The case of Theorem 5.1 when all components of fix $(h)$ are 2-dimensional and orientable is Theorem A of [11]. Case 2 of Theorem 5.2 is the uniqueness result given as Theorem $\mathrm{B}$ of [11].

Proof of Theorem 5.1. We begin by examining vertical actions on $I$-bundles over surfaces of nonnegative Euler characteristic. First, let $A$ be the 1-cell $\{(x, 0) \mid-1 \leq$ $x \leq 1\} \subset\left\{(x, y) \mid x^{2}+y^{2} \leq 1\right\}=D^{2}$. The vertical involution of $D^{2} \times[-1,1]$ that sends $(x, y, t)$ to $(x, y,-t)$ leaves $A \times[-1,1]$ invariant, and we may if we wish regard $D^{2} \times I$ as a product $I$-bundle over $A \times[-1,1]$, so that for this $I$-bundle structure the involution is horizontal. Now consider $S^{1} \times I$ and let $A=S^{1} \times\{1 / 2\}$. Again, the vertical involution of $\left(S^{1} \times I\right) \times[-1,1]$ that sends $((\theta, s), t)$ to $((\theta, s),-t)$ may be regarded as a horizontal involution of a product $I$-bundle over $A \times[-1,1]$. Finally, for the involution of the $I$-bundle over the Möbius band that reflects each $I$-fiber across its midpoint, let $A$ be the Möbius band which is the preimage of the center circle; then the involution can be regarded as a horizontal involution of an $I$-bundle over $A$. Of course, in this case, the original involution was "horizontal." What is accomplished by regarding it as a bundle over $A$ is that $A$ can be chosen to meet the 1-handles in a silvered compression body containing the quotient orbifold of this action.

Let $F_{1}, \ldots, F_{s}$ be the 2-dimensional components of fix $(h)$ that have nonnegative Euler characteristic, and let $G_{1}, \ldots, G_{r}$ be the ones with negative Euler characteristic. As usual, let $p_{1}, \ldots, p_{n}$ denote the isolated fixed points, and $k$ the number of 1-handles of $H / h$. We consider several cases.

Case I: $s \geq 1, r=n=0$, and $k=s-1$.

We will modify the standard construction of $H / h$ given in $\S 2$. In each $F_{i} \times I$, let $a_{i}$ be the image of an invariant $A_{i}$ as defined in the previous paragraph. Note that $a_{i}$ meets each of $F_{i} \times\{0\}$ and $F_{i} \times\{1\}$ in a proper arc, if $F_{i}$ is a disc, or in a center circle, if $F_{i}$ is an annulus or Möbius band. Choose an imbedded disc $D_{1}$ in $F_{1} \times\{1\}$ that meets $a_{1}$ in a proper arc, in $F_{i} \times\{1\}$ for $2 \leq i \leq s-1$ fix disjoint imbedded discs $D_{i}^{\prime}$ and $D_{i}$ each of which meets $a_{i}$ in a proper arc, and fix an imbedded disc $D_{s}^{\prime}$ in $F_{s} \times\{1\}$ that meets $a_{s}$ in a proper arc. Attach 1-handles $K_{i}$ connecting $D_{i}$ to $D_{i+1}^{\prime}$ for $1 \leq i \leq s-1$, and in each $K_{i}$ choose a band $b_{i}$ connecting $D_{i} \cap a_{i}$ to $D_{i+1}^{\prime} \cap a_{i+1}$. The preimage of $\left(\bigcup_{i=1}^{s} a_{i}\right) \cup\left(\bigcup_{i=1}^{s-1} b_{i}\right)$ is a surface $A$ in $H$ such that $H$ can be regarded as an $I$-bundle over $A$ on which $h$ acts horizontally. Note that $A$ is orientable if and only if no $F_{i}$ is a Möbius band.

Case II: $s \geq 1, r=n=0, k \geq s$, and $H / h$ is nonexceptional. 
Proceeding as in Case I, the preimage of $\left(\bigcup_{i=1}^{s} F_{i} \times I\right) \cup\left(\bigcup_{i=1}^{s-1} K_{i}\right)$ is an invariant handlebody $H^{\prime}$ in $H$, containing the preimage $A^{\prime}$ of $\left(\bigcup_{i=1}^{s} a_{i}\right) \cup\left(\bigcup_{i=1}^{s-1} b_{i}\right)$. Now fix a disc $D$ in $F_{1} \times\{1\}$ disjoint from $D_{1}$ and meeting $a_{1}$ in a proper arc, and in $D$ fix $2(k+1-s)$ disjoint discs $D_{i, 1}, D_{i, 2}, s \leq i \leq k$, each meeting $a_{1}$ in a proper arc. For $s \leq i \leq k$ let $K_{i}=D^{1} \times D^{2}$ be a 1-handle with $\{0\} \times D^{2}$ identified with $D_{i, 1}$ and $\{1\} \times D^{2}$ identified with $D_{i, 2}$, so that the union of the $K_{i}$ with a regular neighborhood of $D$ is orientable. In each $K_{i}$ let $b_{i}$ be a band connecting $D_{i, 1} \cap a_{1}$ to $D_{i, 2} \cap a_{1}$. If $a_{1}$ is orientable, choose the $b_{i}$ so that $a_{1} \cup b_{i}$ is still orientable. The preimage $A$ of $\left(\bigcup_{i=1}^{s} a_{i}\right) \cup\left(\bigcup_{i=1}^{k} b_{i}\right)$ is a surface $A$ in $H$ such that $H$ can be regarded as an $I$-bundle over $A$ on which $h$ acts horizontally.

Case III: $s \geq 1, r=n=0, k \geq s$, and $H / h$ is exceptional.

Suppose $H / h$ is exceptional, so that $K_{s}$ is orientation-reversing. Since no $F_{i}$ is a Möbius band, then the band $b_{s}$ can still be chosen so that $A$ is orientable, by making $a_{1} \cup b_{s}$ nonorientable. To see this, consider the example when $s=k=1$ and $F_{1}$ is a disc. Then $a_{1} \cup b_{s}$ is a Möbius band, and its preimage is a punctured torus on which $h$ acts with fixed-point set a nonseparating arc. In fact, this example imbeds in any example, as the preimage of $\alpha \times I \cup b_{s}$ where $\alpha$ is an arc in $\partial a_{1}$ such that $\alpha \times\{1\}$ contains the $\operatorname{arcs} D_{s, 1} \cap a_{1}$ and $D_{s, 2} \cap a_{1}$.

(For the reader who is used to working with orbifold fundamental groups, the algebraic picture in this case is as follows. The union $a=\left(\bigcup_{i=1}^{s} a_{i}\right) \cup\left(\bigcup_{i=1}^{k} b_{i}\right)$ is a 2-orbifold such that $\pi_{1}^{\text {orb }}(a) \rightarrow \pi_{1}^{\text {orb }}(H / h)$ is an isomorphism. Selecting the band $b_{s}$ to reverse orientation in $a$ makes this isomorphism respect the orientation homomorphism. Then, the preimage $A$ of $a$ in $H$ is the orientable double covering of $a$.)

Case IV: $s \geq 1, n=0$, and $r \geq 1$.

In each $G_{j}$, choose a disc $G_{j}^{\prime}$ imbedded in $G_{j}$ that meets $\partial G_{j}$ in an arc, and discs $Z_{j}$ in $F_{1}$ (disjoint from all previously selected discs) so that $Z_{j}$ intersects $a_{1}$ in a proper arc. Attach 1-handles $L_{i}$ connecting $G_{j}^{\prime} \times\{1\}$ to $Z_{j} \times\{1\}$. As above, the preimage $H_{0}$ of $\left(\bigcup_{i=1}^{s} F_{i} \times I\right) \cup\left(\bigcup_{j=1}^{r} G_{j}^{\prime} \times I\right) \cup\left(\bigcup_{i=1}^{k-r} K_{i}\right) \cup\left(\bigcup_{i=1}^{r} L_{i}\right)$ is an $I$ bundle over a 2-manifold $A$ with $h$ acting horizontally. Letting $H_{j}$ be the preimage of $\overline{G_{j}-G_{j}^{\prime}} \times I$ for $1 \leq j \leq r$ yields the theorem.

Case V: $s=n=0$, and $r \geq 1$.

Let $F_{1}$ be a disc imbedded in $G_{1}$ and meeting $\partial G_{1}$ in an arc, and proceed as in Case IV for $2 \leq j \leq r$.

Case VI: $n \geq 1$ and fix $(h)$ contains a component of nonnegative Euler characteristic.

Referring to the standard construction, the preimage of $\left(\bigcup_{j=1}^{n} W_{j}\right) \cup\left(\bigcup_{j=1}^{n-1} L_{j}\right)$ is an invariant handlebody $Z_{n}$ in $H$ of genus $n-1$ on which the restriction of $h$ acts with $n$ isolated fixed points, so has the structure described in $\S 4$. That is, the action is a product action on $S_{n} \times I$.

We may choose notation so that $F_{m}$ has nonnegative Euler characteristic. By the previous five cases, the preimage of $F_{m} \times I$ is contained in an invariant handlebody $H_{0}^{\prime}$, an $I$-bundle over a 2-manifold $A$ on which the restriction of $h$ acts horizontally, and $F_{m} \times I$ contains a portion $a_{m}$ of the image of $A$. We may perform the standard construction so that $E_{1}^{\prime}$ meets the image of $S_{n} \times\{1 / 2\}$ in an arc, and $D_{m}$ meets $a_{m}$ in an arc. Then, we choose a band in $L_{n}$ that connects these arcs. The invariant $I$-fiberings of $H_{0}^{\prime}$ and $Z_{n}$ define $I$-fiberings of their images, and these extend to an $I$-fibering of the union of the images with $L_{n}$. Therefore the union of $H_{0}^{\prime}, Z_{n}$, 
and the preimage of $L_{n}$ has an invariant $I$-fibering, and this union is the $H_{0}$ in Theorem 5.1.

Case VII: $n \geq 1$ and fix $(h)$ contains no component of nonnegative Euler characteristic.

Let $F_{1}$ be a disc imbedded in $G_{1}$ and meeting $\partial G_{1}$ in an arc, and proceed as in Case VI. This completes the proof of Theorem 5.1.

Remark 5.5. When fix $(h)$ has isolated points in addition to 2-dimensional components, the invariant $I$-bundle structure is never a product bundle, since the action of $h$ on $H_{0}$ has both 0 - and 2-dimensional components in its fixed-point set.

Proof of Theorem 5.2. Let $h_{j}$ denote the restriction of $h$ to $H_{j}$ for $1 \leq j \leq r$. By property (ii), each $h_{j}$ has fixed-point set a deformation retract of one of the components of fix $(h)$ which is a surface with negative Euler characteristic. Therefore the sets fix $\left(h_{j}\right)$ are determined up to homeomorphism. Since the $h_{j}$ are vertical, they are determined up to equivalence.

Let $h_{0}$ denote the restriction of $h$ to $H_{0}$. Each disc of $H_{0} \cap H_{j}$ must meet a disc component of fix $\left(h_{0}\right)$ that lies in a component of fix $(h)$ of negative Euler characteristic. Thus there are $r$ components of fix $\left(h_{0}\right)$ that are discs contained in the $r$ components of fix $(h)$ of negative Euler characteristic. The remaining components of fix $\left(h_{0}\right)$ must be exactly the components of fix $(h)$ that have nonnegative Euler characteristic. Therefore fix $(h)$ and fix $\left(h_{0}\right)$ have the same number of components, and fix $\left(h_{0}\right)$ is uniquely determined up to homeomorphism. Also, each fix $\left(h_{j}\right)$ contributes equally to the ranks of $H_{1}(H)$ and $H_{1}(\operatorname{fix}(h))$, so $\chi(\operatorname{fix}(h))-\chi(H)=\chi\left(\operatorname{fix}\left(h_{0}\right)\right)-\chi\left(H_{0}\right)$.

If every component of fix $(h)$ has nonnegative Euler characteristic, then $H=H_{0}$ and $h=h_{0}$. If every component of fix $(h)$ is orientable, then $\left|H_{0} / h_{0}\right|$ is orientable if and only if $|H / h|$ is; hence by Proposition $3.5, h_{0}$ is determined up to equivalence by $h$. If some component of fix $(h)$ is a Möbius band, then $\left|H_{0} / h_{0}\right|$ is nonorientable and again $h_{0}$ is determined. If $\operatorname{fix}(h)$ has more than $(\chi(\operatorname{fix}(h))-\chi(H)) / 2$ components, then the number of components of fix $\left(h_{0}\right)$ exceeds $\left(\chi\left(\operatorname{fix}\left(h_{0}\right)\right)-\chi\left(H_{0}\right)\right) / 2$, so by Theorem $3.6 h_{0}$ is uniquely determined.

Suppose now that none of the four conditions holds. Then every component of fix $\left(h_{0}\right)$ of nonnegative Euler characteristic is 2-dimensional and orientable, but some component of fix $(h)$, say $N_{1}$, is nonorientable. Referring to the proof of Theorem 5.1, denote by $H^{\prime}$ the handlebody in $H / h$ which is a regular neighborhood of $D \cup\left(\bigcup_{i=s}^{k-r} K_{i}\right)$. Since $r+s$ (the number of components of fix $\left.\left(h_{0}\right)\right)$ does not exceed $\left(\chi\left(\operatorname{fix}\left(h_{0}\right)\right)-\chi\left(H_{0}\right)\right) / 2$ (the number of 1-handles of $H_{0} / h_{0}$, by the Riemann-Hurwitz Formula), it follows that $s \leq k-r$, so $H^{\prime}$ has at least one 1-handle. Since each component of fix $\left(h_{0}\right)$ is orientable, $\left|H_{0} / h_{0}\right|$ is orientable if and only if $H^{\prime}$ is. Since $N_{1}$ is nonorientable, however, there is a homeomorphism from $H / h$ to another silvered compression body with the same structure except that the orientability of $H^{\prime}$ is changed. This homeomorphism can be described as "sliding" the attaching discs for the 1-handles in $H^{\prime}$ around orientation-reversing loops in the boundary of the closure of $|H / h|-\bigcup_{i=s}^{k-r} K_{i}$. Therefore the decomposition of $H / h$ in the proof of Theorem 5.1 can be chosen with either orientability of $\left|H_{0} / h_{0}\right|$, and hence with $h_{0}$ being either of the possible orientation-reversing involutions on $H_{0}$.

Our second decomposition isolates the part of the action with 0-dimensional fixed-point set, and also forces the action on $H_{0}$ to be a product action. When fix $(h)$ contains isolated fixed points, it requires cutting along one pair of discs 
interchanged by $h$, rather than only along invariant discs as in the vertical-horizontal decomposition.

Theorem 5.6 (Product decomposition). Let $h$ be an orientation-reversing involution of an orientable handlebody $H$. Assume that fix $(h)$ contains a 2-dimensional component $F_{1}$, and has $n \geq 0$ isolated fixed points. Then $H$ has a decomposition $H=H_{0} \cup\left(\bigcup_{j=1}^{r} H_{j}\right) \cup Z_{n}$, where each piece is $h$-invariant, such that

(i) $H_{0}$ is a product I-bundle over a connected 2-manifold $A$, and the restriction of $h$ to $H_{0}$ is horizontal.

(ii) Each $H_{j}$ is an I-bundle over a Möbius band or a surface of negative Euler characteristic, which is a deformation retract of a component of fix $(h)$, and the restriction of $h$ to $H_{j}$ is vertical.

(iii) If $n=0$, then $Z_{n}$ is empty; otherwise $Z_{n}$ has genus $n-1$ and the restriction of $h$ to $Z_{n}$ is a product action with fixed-point set consisting of $n$ isolated fixed points.

(iv) $\left\{Z_{n}, H_{1}, \ldots, H_{r}\right\}$ are pairwise disjoint, each of $H_{0} \cap H_{i}, 1 \leq i \leq r$, is a single 2 -disc, and if $n \geq 1$ then $H_{0} \cap Z_{n}$ consists of two 2-discs interchanged by $h$.

Theorem 5.7. Let $h$ be an orientation-reversing involution of a handlebody $H$, and suppose that some component of $\operatorname{fix}(h)$ is 2-dimensional. In any product decomposition $H=H_{0} \cup\left(\bigcup_{j=1}^{r} H_{j}\right) \cup Z_{n}$ satisfying conditions (i), (ii), (iii), and (iv) in Theorem 5.6, the actions on the $H_{j}$ for $j \geq 1$ are determined up to equivalence (and ordering of the indices). The action on $H_{0}$ is determined up to equivalence if any of the following holds:

1. Every component of fix $(h)$ has nonnegative Euler characteristic.

2. Every component of $\operatorname{fix}(h)$ is 2-dimensional and orientable.

3. Some component of fix $(h)$ is a point.

4. The number of components of fix $(h)$ exceeds $(\chi(\operatorname{fix}(h))-\chi(H)) / 2$.

In the remaining cases, either of two possible actions on $H_{0}$ can appear in product decompositions of $h$.

Proof of Theorems 5.6 and 5.7. The proofs are almost exactly like the proofs of Theorems 5.1 and 5.2. For Theorem 5.6, regard any Möbius band components of fix $(h)$ as $G_{i}$ 's rather than as $F_{i}$ 's, and in Cases VI and VII do not recombine $Z_{n}$ with $H_{0}^{\prime}$.

The decomposition theorems yield elegant characterizations of fiber-preserving actions. The first corollary generalizes Theorem 3.1 of [10].

Corollary 5.8. Let $h$ be an orientation-reversing involution of a handlebody $H$. Then, in some coordinates, $H$ is of the form $A \times I$ on which $h$ acts preserving I-fibers if and only if one of the following holds.

(i) $h$ is a vertical involution of a product I-bundle, or

(ii) every component of fix $(h)$ is a disc or annulus, or

(iii) every component of $\operatorname{fix}(h)$ is a point.

Proof. The only if direction is immediate from the observations at the end of $\S 1$. Conversely, if $h$ is a vertical involution of a product $I$-bundle, there is nothing to show. If every component of $\operatorname{fix}(h)$ is a disc or annulus, then the Product Decomposition Theorem shows that $h$ is a horizontal involution of a product $I$-bundle. If every component of $\operatorname{fix}(h)$ is a point, then the result follows from Theorem 4.2. This completes the proof. 
Allowing components of the fixed point set to be Möbius bands gives a generalization of Theorem 3.1' of Nelson [10].

Corollary 5.9. Let $h$ be an orientation-reversing involution of a handlebody $H$. Then in some coordinates, $H$ is an I-bundle on which $h$ acts preserving I-fibers if and only if one of the following holds.

(i) $h$ is a vertical involution of an I-bundle, or

(ii) every component of fix $(h)$ has nonnegative Euler characteristic.

Proof. The only if direction is immediate from the observations at the end of $\S 1$. Conversely, if $h$ is a vertical involution of an $I$-bundle, there is nothing to show. If every component of fix $(h)$ has nonnegative Euler characteristic and at least one is 2 -dimensional, then the Vertical-Horizontal Decomposition Theorem shows that $h$ is a horizontal involution of an $I$-bundle. If every component of $\operatorname{fix}(h)$ is a point, then the result follows from Theorem 4.2. This completes the proof.

Our last corollary extends Theorem 3.2 of Nelson [10].

Corollary 5.10. Let $h$ be an orientation-reversing involution of a handlebody $H$. Then $\chi(\operatorname{fix}(h))=\chi(H)$ if and only if either $h$ is the antipodal map of the 3-ball, or $h$ is the free involution on the solid torus, or $h$ is a vertical involution of an I-bundle.

Proof. By the Riemann-Hurwitz Formula, $k=0$, so fix $(h)$ is connected. If $H$ is the ball, then $\operatorname{fix}(h)$ is a point or a disc and $h$ is either the antipodal map or the vertical involution of $D^{2} \times I$. If $H$ is the solid torus, then $\chi(\operatorname{fix}(h))=0$ only when fix $(h)$ is empty, in which case $h$ is free, or $\operatorname{fix}(h)$ is an annulus or a Möbius band and $h$ is vertical. If $H$ has genus at least two, then fix $(h)$ has a single component which is a surface of negative Euler characteristic. In the Vertical-Horizontal Decomposition Theorem, $H_{0}$ must be $D^{2} \times I$ and $H_{0} \cup H_{1}$ is an $I$-bundle on which $h$ acts as a vertical involution.

Remark 5.11. Roughly speaking, the vertical-horizontal decomposition makes the horizontal part as large as possible (while keeping the decomposition reasonably natural), while the product decomposition makes the parts with product action as large as possible. As we mentioned at the beginning of the section, there are several other ways to decompose orientation-reversing involutions. Perhaps the most natural of these approaches is to try to pick out as many vertical actions as possible. Arguments similar to the proofs given above yield the following.

Theorem 5.12 (Maximal Vertical Decomposition). Let $h$ be an orientationreversing involution of an orientable handlebody $H$. Assume that fix $(h)$ contains a 2-dimensional component $F_{1}$, and has $n \geq 0$ isolated fixed points. Then $H$ has a decomposition $H=H_{0} \cup\left(\bigcup_{j=1}^{r} H_{j}\right) \cup Z_{n}$, where each piece is $h$-invariant, such that

(i) $H_{0}$ is an I-bundle over a connected 2-manifold $A$, the restriction of $h$ to $H_{0}$ is horizontal, and each component of fix $(h)$ has positive Euler characteristic. This may be chosen to be a product action if and only if no component of $\operatorname{fix}(h)$ is a point.

(ii) Each $H_{j}$ is an I-bundle over a surface of nonpositive Euler characteristic, which is a deformation retract of a component of fix $(h)$, and the restriction of $h$ to $H_{j}$ is vertical.

(iii) $\left\{H_{1}, \ldots, H_{r}\right\}$ are pairwise disjoint, and for $1 \leq i \leq r$ each $H_{0} \cap H_{i}$ is a single 2-disc. 
Theorem 5.13. Let $h$ be an orientation-reversing involution of a handlebody $H$, and suppose that some component of $\mathrm{fix}(h)$ is 2-dimensional. In any maximal vertical decomposition

$$
H=H_{0} \cup\left(\bigcup_{j=1}^{r} H_{j}\right)
$$

satisfying conditions (i), (ii), and (iii) in Theorem 5.12, the actions on the $H_{j}$ for $j \geq 1$ are determined up to equivalence (and ordering of the indices). The action on $H_{0}$ is determined up to equivalence if any of the following holds:

1. Every component of fix $(h)$ has positive Euler characteristic.

2. Every component of $\mathrm{fix}(h)$ is 2-dimensional and orientable.

3. Some component of fix $(h)$ is a point.

4. The number of components of fix $(h)$ exceeds $(\chi(\operatorname{fix}(h))-\chi(H)) / 2$.

In the remaining cases, either of two possible actions on $H_{0}$ can appear in verticalhorizontal decompositions of $h$.

One can modify the maximal vertical decomposition by splitting off the 0dimensional part of fix $(h)$, as was done to obtain the product decomposition from the vertical-horizontal decomposition. A minor modification of the product decomposition is obtained by amalgamating the vertical actions which have fixed-point set a Möbius band into the $H_{0}$ (as is done in the vertical-horizontal decomposition). There are also more drastic approaches where one makes the horizontal part as large as possible by amalgamating portions of the vertical actions in the vertical-horizontal decomposition into the horizontal part. Since these split up the components of fix $(h)$ that have negative Euler characteristic, they are rather artifical and lead to more complicated uniqueness statements.

\section{REFERENCES}

1. F. Bonahon, Cobordisme des difféomorphisms des surfaces, C. R. Acad. Sci. Paris Sér. A 290 (1987), 765-767. MR 81d:57028

2. P.E. Conner and F. Raymond, Actions of compact Lie groups on aspherical manifolds, Topology of Manifolds (Proc. Inst., Univ. of Georgia, Athens Ga., 1969), Markham, Chicago, 1970, pp. 227-264. MR 42:6839

3. M. Davis and J. Morgan, Finite group actions on homotopy spheres, The Smith Conjecture (J. Morgan and H. Bass, eds.), Academic Press, Orlando, 1984, pp. 181-226. MR 86i:57002

4. P.K. Kim and J. Tollefson, Splitting the PL involutions of nonprime 3-manifolds, Michigan Math. J. 27 ( (1980), 259-274. MR 81m:57007

5. P.K. Kim and J. Tollefson, PL involutions of fibered 3-manifolds, Trans. Amer. Math. Soc. 232 (1974), 221-237. MR 56:13223

6. D. McCullough and A. Miller, Homeomorphisms of 3-manifolds with compressible boundary, Mem. Amer. Math. Soc. 344 (1986), 1-100. MR 87i:57013

7. D. McCullough, A. Miller, and B. Zimmermann, Group actions on handlebodies, Proc. London Math. Soc. 59 (1989)), no. 3, 373-416. MR 90h:57014

8. W. Meeks and P. Scott, Finite group actions on 3-manifolds, Invent. Math. 86 (1986), 287346. MR 88b:57039

9. W. Meeks and S-T. Yau, The equivariant Dehn's lemma and loop theorem, Comment. Math. Helv. 56 (1981), 225-239. MR 83b:57006

10. R. Nelson, Some fiber-preserving involutions of orientable 3-dimensional handlebodies, Houston J. Math. 9 (1983), 255-269. MR 84k:57029

11. R. Nelson, A unique decomposition of involutions of handlebodies, Proc. Amer. Math. Soc. 93 (1985), 358-362. MR 86g:57031 
12. M. Stephanus, Orientation reversing involutions of a handlebody, Dissertation at St. Louis University (1995).

13. W. Thurston, The geometry and topology of 3-manifolds, mimeographed notes, Princeton University.

Department of Mathematics, St. Louis University, St. Louis, Missouri 63103

E-mail address: kalliongisje@sluvca.slu.edu

Department of Mathematics, University of Oklahoma, Norman, Oklahoma 73019

E-mail address: dmccullough@uoknor.edu 